\title{
ARTICLE Association between irritability and suicidal ideation in three clinical trials of adults with major depressive disorder
}

\author{
Manish K. Jha ${ }^{1,2}$, Abu Minhajuddin ${ }^{2}$, Cherise Chin Fatt ${ }^{2}$, Katharina Kircanski ${ }^{3}$, Argyris Stringaris ${ }^{3}$, Ellen Leibenluft ${ }^{3}$ and \\ Madhukar H. Trivedi iD $^{2}$
}

Irritability in pediatric samples is associated with higher rates of subsequent suicide-related outcomes. No study, to date, has evaluated the longitudinal association between irritability and suicidal ideation (SI) in adults with major depressive disorder (MDD). This report evaluated whether irritability is associated with SI at the same visit (i.e., concurrently) and whether early changes in irritability with antidepressant treatment predict subsequent levels of SI. Participants of Combining Medications to Enhance Depression Outcomes (CO-MED, $n=665$ ), Establishing Moderators and Biosignatures of Antidepressant Response in Clinical Care (EMBARC, $n=296$ ), and Suicide Assessment Methodology Study (SAMS, $n=266$ ) were included. Repeated-measures mixed model analyses evaluated concurrent association throughout the trial between irritability (five-item irritability domain of Concise Associated Symptom Tracking scale) and SI (three-item suicidal thoughts factor of Concise Health Risk Tracking scale) after controlling for overall depression (excluding suicidality-related item), and predicted subsequent levels of SI (repeated observations from week-2-to-week-8) based on early (baseline-to-week-2) changes in irritability after controlling for early changes in overall depression. Higher irritability was associated with higher SI concurrently; estimates (standard error) were $0.18(0.02, p<0.0001), 0.64$ $(0.02, p<0.0001)$, and $0.26(0.04, p<0.0001)$ in CO-MED, EMBARC, and SAMS respectively. Greater baseline-to-week- 2 reductions in irritability predicted lower levels of subsequent Sl; estimates (standard errors) were $-0.08(0.03, p=0.023),-0.50(0.05, p<0.0001)$, and $-0.12(0.05, p=0.024)$ in CO-MED, EMBARC, and SAMS, respectively. Controlling for anxiety or insomnia produced similar results. In conclusion, irritability and SI were consistently linked in adults with MDD. These findings support careful assessment of irritability in suicide risk assessment.

Neuropsychopharmacology (2020) 45:2147-2154; https://doi.org/10.1038/s41386-020-0769-x

\section{INTRODUCTION}

Increasing rates of suicide-related mortality have underscored the urgency to identify reliable and potentially modifiable risk factors of suicidality [1]. While depression severity has been wellrecognized as a risk factor for suicide [2-4], irritability has gained recent attention as an independent risk factor [5]. Longitudinal studies of youths have linked (1) symptoms of irritability to suicidal ideation (SI) concurrently (i.e., at the same visit) [6]; (2) symptoms of irritability during childhood [7] or adolescence [8] to increased likelihood of suicide attempts later in life; and (3) the presence of irritability prior to treatment initiation to occurrence of suicide-related events (SI, self-mutilation and suicide attempt) during the first three months of antidepressant treatment [9]. One longitudinal study of adults with bipolar or schizoaffective disorder found that the presence of irritability within the context of manic, depressive, mixed, subsyndromal, or euthymic presentation at index visit was associated with higher severity of depressive symptoms, including $\mathrm{SI}$, over a two-year naturalistic observation period [10]. A recent longitudinal study that followed offspring of parents with mood disorders for up to 12 years reported that elevated trajectory of irritability was associated with higher likelihood of suicide attempt [11]. Furthermore, the presence of irritability in adults who were assessed in emergency room for SI or suicide attempt was associated with higher likelihood of suicide behavior at 12 months of follow-up [12]. Finally, a recent study of patients with Huntington's disease who were followed for up to 5 years reported that higher frequency of irritable behaviors was associated with shorter time to occurrence of SI or suicide attempt [13]. However, to date no study has dissociated the effects of treatment on levels of irritability, depression, and $\mathrm{SI}$ in adults with MDD, and examined whether reduction in irritability with treatment is associated with decreased levels of subsequent SI. Here, in the context of two randomized controlled trials (RCTs) and one open trial of patients treated for major depressive disorder (MDD) (total $n=1227$ ), we examine associations among response to treatment of irritability, depression, and SI (active suicidal thoughts and plans).

When examining associations between irritability and $\mathrm{SI}$, it is particularly important to dissociate effects of depression versus irritability on SI. A large cross-sectional study of adult outpatients with MDD found that the association of irritability with current SI and lifetime history of suicide attempt was no longer significant

\footnotetext{
${ }^{1}$ Department of Psychiatry, Icahn School of Medicine at Mount Sinai, 1 Gustave L. Levy PI, New York, NY 10029, USA; ${ }^{2}$ Center for Depression Research and Clinical Care, UT Southwestern Medical Center, 5323 Harry Hines Blvd., Dallas, TX 75390-9119, USA and ${ }^{3}$ National Institute of Mental Health, 10 Center Dr, Bethesda, MD 20814, USA Correspondence: Madhukar H. Trivedi (madhukar.trivedi@utsouthwestern.edu)

These authors contributed equally: Manish K. Jha, Abu Minhajuddin

Findings of this report were presented in part at the 2019 Annual Meeting of American College of Neuropsychopharmacology.
}

Received: 13 May 2020 Revised: 19 June 2020 Accepted: 8 July 2020

Published online: 14 July 2020 
after adjusting for age, sex, and overall depression severity [14]. Consistent with this, other studies of adults with depression did not find a significant cross-sectional association between irritability and SI after accounting for potential confounders such as anxiety and insomnia [5, 15-17].

Understanding the association of irritability with $\mathrm{SI}$ will also inform the utility of irritability as an important target symptom [18]. While irritability is not a diagnostic criterion for MDD in adults [19], it reported by about half of adults with $\operatorname{MDD}[14,20]$ and is associated with greater illness severity and worse long-term clinical course [14, 20,21]. Furthermore, we have previously found (using data from one RCT and one open label trial also included in this report) that early changes in irritability independently predict subsequent levels of overall depression [22]. However, our previous report [22] did not assess associations between the response of irritability to treatment and alterations in SI.

Thus, in this report, we seek to systematically assess the association of irritability with SI while accounting for the severity of other depressive symptoms (referred to as overall depression hereafter) [23], using three samples of convenience that used the same instruments to measure irritability and SI. The two RCTs, Combining Medications to Enhance Depression Outcomes (COMED) and Establishing Moderators and Biosignatures of Antidepressant Response in Clinical Care (EMBARC) enrolled $n=665$ and $n=296$ patients with MDD, respectively. The only open trial, Suicide Assessment Methodology Study (SAMS), enrolled $n=266$ patients with MDD for eight-week long treatment with a selective serotonin reuptake inhibitor (SSRI). Using data from these three trials, we asked the following questions regarding irritability and SI:

(1) Is irritability associated with SI concurrently?

(2) If yes, is this association significant even after controlling for overall depression?

(3) Do early changes in irritability with treatment predict subsequent levels of SI even after controlling for early changes in overall depression?

As the trials differed in their eligibility criteria and treatments, these questions regarding irritability, overall depression and SI were asked separately for each trial. While the trials differed in treatment duration, all included visits up to 8 weeks after baseline; therefore, only visits from baseline-to-week-8 were included in this analysis. Longitudinal study design that assessed irritability and $\mathrm{SI}$ at the same visit and predicted levels of SI at subsequent visits based on early changes in irritability was used to provide a stable estimate of concurrent association and to evaluate whether irritability is a potentially modifiable risk factor for suicidality. Analyses that controlled for overall depression were used to inform the clinical utility of measuring irritability in addition to the existing practice of focusing on overall depression [24]. As anxiety is associated with irritability and insomnia is associated with $\mathrm{SI}$ $[25,26]$, additional analyses that controlled for these symptoms were used to demonstrate the specificity of association between irritability and SI.

\section{METHODS}

Study design, participants, and interventions

All three trials were approved by the Institutional Review Boards at each site and all participants provided signed written informed consent prior to completing any study related procedures. Furthermore, irritability, SI, overall depression, anxiety, and insomnia were assessed at each study visit.

CO-MED. As described previously, from March 2008 to February 2009, the CO-MED trial (NCT00590863) recruited 18-75-year-old treatment-seeking MDD outpatients $(n=665)$, see Supplementary materials for CONSORT diagram and protocol, with at least moderately severe [17-item Hamilton Depression Rating scale $($ HAMD-17) $\geq 16)$ nonpsychotic chronic or recurrent depression from six primary and nine psychiatric sites after obtaining participants' written informed consent and approval of IRB from each participating site [27]. Detailed eligibility criteria are available at https:// clinicaltrials.gov/ct2/show/NCT00590863. Participants were randomly assigned in 1:1:1 ratio stratified by site (using web-based randomization in block sizes of three and six) at baseline to singleblind (clinician unblinded, participant blinded to second medication only) treatment with either escitalopram-plus-placebo, sustainedrelease-bupropion-plus-escitalopram, or extended-release-venlafaxine-plus-mirtazapine.Post-randomization visits were conducted at week-1, $-2,-4,-6,-8,-10$, and -12 for acute phase.

EMBARC. As previously described [28], from August 2011 to December 2015, EMBARC (NCT01407094) enrolled 309 participants with MDD at four academic medical centers, of whom ten were in a feasibility sample and three were randomized but were not eligible, resulting in an intent to treat sample of $n=296$, also see supplementary materials for CONSORT diagram and protocol. The inclusion and exclusion criteria have been described previously $[28,29]$. Briefly, 18-65-year-old participants with at least moderately severe [Quick Inventory of Depressive Symptomatology (QIDS-SR) $\geq 14$ ] depression at both screening and randomization visits who did not meet criteria for any failed antidepressant trial in the current episode based on Massachusetts General Hospital Antidepressant Treatment Response Questionnaire (MGH-ATRQ) were randomized to double-blind (both participant and clinician) treatment with either sertraline or placebo in 1:1 ratio stratified by site (in clinician trial management software) for Stage 1 and assessed at baseline and week-1, -2, -3, $-4,-6$, and -8 .

SAMS. As described previously, SAMS recruited 18-75-year-old treatment-seeking outpatients $(n=266)$ with nonpsychotic MDD and moderately severe depression (HAMD-17 $\geq 14$ ) from six primary and nine psychiatric care sites [30, 31]. Detailed eligibility criteria are available at https://clinicaltrials.gov/ct2/show/ NCT00532103. Participants received a SSRI medication (escitalopram, citalopram, sertraline, paroxetine, paroxetine controlled release, or fluoxetine) in an open label fashion for 8 weeks (postbaseline visits at week-2, $-4,-6$, and -8 ).

\section{Measurements}

Irritability. In all three trials, participants completed the five-item irritability domain of the Concise Associated Symptom Tracking scale (CAST-IRR) as a measure of irritability at each visit. Individual items were "I wish people would just leave me alone," "I feel very uptight," "I find myself saying or doing things without thinking," "Lately everything seems to be annoying me," and "I find people get on my nerves easily." These items were rated on a five-point Likert scale with responses of "strongly disagree," "disagree," "neither agree nor disagree," "agree," and "strongly agree" corresponding to scores of 1, 2, 3, 4, and 5 (total score range: 5-25). Cronbach's alpha of CAST-IRR at baseline in CO-MED, EMBARC, and SAMS were 0.83, 0.77, and 0.78, respectively. Previous reports have found strong associations of CAST-IRR with measures of anger and irritability (convergent validity) and minimal associations with measures of mania, insomnia, and anhedonia (divergent validity), underscoring strong psychometric properties of CAST-IRR [31-33]. A modified score of irritability was computed after excluding the impulsivity-related item, "I find myself saying or doing things without thinking."

Depression. Clinicians conducted structured interviews [34] for the 17-item Hamilton Depression Rating Scale (HAMD) [35] to assess depression severity at each visit of the EMBARC, and the 
16-item Quick Inventory of Depressive Symptomatology ClinicianRated (QIDS-C) [36] at each visit of the CO-MED and SAMS trials. Previous reports have found high concurrent validity of HAMD and QIDS-C with other measures of depression severity such as the 30-item Inventory of Depressive Symptomatology [36, 37]. The Quick Inventory of Depressive Symptomatology Self-Report version (QIDS-SR) [36] was collected prior to treatment initiation only in EMBARC and at each visit of CO-MED trial. In addition, HAMD was collected prior to treatment initiation only in CO-MED and in SAMS trials. In this report, HAMD in EMBARC and QIDS-C in CO-MED and SAMS trials were the measures of overall depression severity (all clinician-rated) after removal of suicidality-related item.

Suicidal ideation. Participants completed the three-item suicidal thoughts factor of the Concise Health Risk Tracking (CHRT) as a measure of SI at each visit in all three trials [38]. These items included, "I have been having thoughts of killing myself," "I have thoughts about how I might kill myself," and "I have a plan to kill myself." These items were rated on a five-point Likert scale with responses of "strongly disagree," "disagree," "neither agree nor disagree," "agree," and "strongly agree" corresponding to scores of $0,1,2,3$, and 4 , respectively (total score range: $0-12$ ).

Anxiety. Participants completed the three-item anxiety domain of CAST-ANX as a measure of anxiety at each visit. Individual items included, "I feel anxious all the time," "I am feeling restless, as if I have to move constantly", and "I cannot sit still." These items were rated on a five-point Likert scale with responses of "strongly disagree," "disagree," "neither agree nor disagree," "agree," and "strongly agree" corresponding to scores of 1, 2, 3, 4, and 5 (total score range: $3-15)$. Previous reports have found strong psychometric properties of CAST-ANX [32, 33].

Insomnia. Participants completed the two-item anxiety domain of CAST-INS as a measure of insomnia at each visit. Individual items included, "I have been having more trouble sleeping than usual" and "I slept very little last night". These items were rated on a five-point Likert scale with responses of "strongly disagree," "disagree," "neither agree nor disagree," "agree," and "strongly agree" corresponding to scores of 1, 2, 3, 4, and 5 (total score range: $2-10)$. Previous reports have found strong psychometric properties of CAST-INS [32, 33].

\section{Statistical analysis plan}

Descriptive statistics were used to describe the analytic sample for this report and to present the observed levels of irritability, suicidality, and overall depression at each visit. Age, sex, race, and ethnicity were used as covariates in all analyses. All symptom measures were standardized ( $z$ transformed with mean of 0 and standard deviation of 1 ) to allow for comparison of $\beta$ estimates given that individual symptom measures differed in their range of total scores.

Repeated-measures correlation coefficients [39] were estimated separately in each trial to assess the concurrent association between irritability and SI across all available time points. For each trial separately, repeated-measures mixed model analyses (compound symmetry covariance structure) were used with irritability and overall depression as key independent variables of interest in the same model and $\mathrm{SI}$ as the outcome variable to evaluate whether concurrent association of irritability with SI was significant even after controlling for levels of overall depression at the same visit.

To evaluate whether early changes in irritability significantly predicted subsequent suicidality, repeated-measures mixed model analyses (compound symmetry covariance structure) were used with repeated observation of SI at each available visit (from week2-to-week-8) as the outcome variable and baseline-to-week-2 changes in irritability and overall depression as key independent variables of interest. Additional covariates for these models included baseline levels of irritability, overall depression, and SI.

Additional analyses substituted anxiety or insomnia for overall depression in the aforementioned mixed model analyses to test whether association between irritability and SI was significant even after controlling for these symptoms. Additional analyses, in which a modified irritability scale was substituted for CAST-IRR, were conducted to evaluate: (1) correlation coefficient between the modified irritability measure and $\mathrm{Sl}$; $(2)$ concurrent association between the modified irritability measure and SI from baseline-toweek-8 in a repeated-measures mixed model analysis that controlled for either overall depression, anxiety or insomnia (Supplementary Table S4); and (3) association between baselineto-week-2 change in the modified irritability measure and SI from week-2-to-week-8 in a repeated measures mixed model analysis that controlled for baseline-to-week-2 change in overall depression (Supplementary Table S7).

To evaluate whether the association between irritability and SI was specific to antidepressant medications, we conducted analyses that were stratified by treatment arm (sertraline and placebo) in EMBARC. Specifically, for sertraline and placebo arms separately, we conducted (1) mixed model analyses (including data from all visits from baseline-to-week-8) with irritability and overall depression as key independent variables of interest in the same model and SI as the outcome variable; and (2) mixed model analyses with repeated observations of $\mathrm{SI}$ at each available visit (from week-2-to-week-8) as the outcome variable and baseline-toweek-2 changes in irritability and overall depression as key independent variables of interest.

Given the nature of these unplanned secondary analyses, no formal sample size estimation was done and sample size was restricted by availability of data in each study. All analyses were conducted using SAS 9.4 and the threshold of significance was set at $p<0.05$ without any adjustment for multiple comparisons.

\section{RESULTS}

At baseline, the three trials [CO-MED $(n=665)$, EMBARC $(n=296)$, and SAMS $(n=266)]$ were similar on most clinical and demographic characteristics, except that the proportion of unemployed participants was higher in CO-MED (50.2\%) than in SAMS (35.8\%), and participants in SAMS reported lower levels of suicidality than in CO-MED or in EMBARC, see Table 1. Observed means and standard deviations of irritability, suicidality, and overall depression are presented in Fig. 1.

Is irritability associated with SI concurrently?

Yes. In repeated-measures analyses that estimated concurrent association averaged across each available visit, irritability was positively correlated (Pearson's $r=0.31-0.73$ ) with SI in all three trials (all $p<0.0001$ ). See Table 2 for the correlation coefficients and $95 \%$ confidence intervals of irritability, overall depression, anxiety, and insomnia with SI.

Is this association significant even after controlling for overall depression?

Yes. In repeated-measures mixed model analyses with SI as the outcome variable and irritability and overall depression as independent variables of interest in the same model, irritability was significantly associated with SI even after controlling for overall depression; standardized estimates $(\beta)$ [standard errors (SE)] were $0.18(0.02, p<0.0001), 0.64(0.02, p<0.0001)$, and 0.26 $(0.04, p<0.0001)$ in CO-MED, EMBARC, and SAMS respectively; also see Supplementary Table S1. Furthermore, the strength of association between irritability and $\mathrm{SI}$ in these models was significantly higher than that of overall depression and SI in two out of three (EMBARC and SAMS) trials, see Fig. 2. 
Table 1. Baseline clinical and demographic characteristics of CO-MED, EMBARC, and SAMS trials.

\begin{tabular}{|c|c|c|c|c|c|c|}
\hline \multirow{3}{*}{$\begin{array}{l}\text { Number } \\
\text { Categorical variables }\end{array}$} & \multirow{2}{*}{\multicolumn{2}{|c|}{$\frac{\text { CO-MED }}{665}$}} & \multirow{2}{*}{\multicolumn{2}{|c|}{$\frac{\text { EMBARC }}{296}$}} & \multirow{2}{*}{\multicolumn{2}{|c|}{$\frac{\text { SAMS }}{266}$}} \\
\hline & & & & & & \\
\hline & $N$ & $\%$ & $N$ & $\%$ & $N$ & $\%$ \\
\hline \multicolumn{7}{|l|}{ Sex } \\
\hline Male & 213 & 32.03 & 102 & 34.46 & 77 & 28.95 \\
\hline Female & 452 & 67.97 & 194 & 65.54 & 189 & 71.05 \\
\hline \multicolumn{7}{|l|}{ Race } \\
\hline White & 425 & 63.91 & 193 & 65.20 & 181 & 68.05 \\
\hline Black & 176 & 26.47 & 58 & 19.59 & 68 & 25.56 \\
\hline Other & 64 & 9.62 & 45 & 15.20 & 17 & 6.39 \\
\hline Hispanic ethnicity & 101 & 15.19 & 55 & 18.58 & 31 & 11.65 \\
\hline Unemployed at baseline & 334 & 50.23 & 124 & 42.47 & 95 & 35.85 \\
\hline Continuous variables & Mean & SD & Mean & SD & Mean & SD \\
\hline Mean age in years & 42.71 & 13.00 & 37.06 & 13.28 & 41.21 & 13.45 \\
\hline $\begin{array}{l}\text { HAMD- } 17 \text { minus } \\
\text { suicide item }\end{array}$ & 22.86 & 4.55 & 17.74 & 4.25 & 20.28 & 4.26 \\
\hline $\begin{array}{l}\text { QIDS-C minus } \\
\text { suicide item }\end{array}$ & 15.02 & 3.15 & NA & NA & 14.15 & 2.98 \\
\hline CAST Irritability domain & 17.34 & 3.80 & 16.33 & 3.96 & 15.90 & 4.38 \\
\hline $\begin{array}{l}\text { CHRT Suicidal Thoughts } \\
\text { factor }\end{array}$ & 5.09 & 2.65 & 5.37 & 2.35 & 2.02 & 2.56 \\
\hline CAST Anxiety domain & 9.24 & 2.95 & 8.84 & 2.66 & 8.86 & 2.95 \\
\hline CAST Insomnia domain & 7.06 & 2.33 & 6.64 & 2.06 & 7.25 & 2.30 \\
\hline
\end{tabular}

Irritability was assessed with the five-item irritability domain of Concise Associated Symptom Tracking (CAST) scale. Suicidal ideation was assessed with three-item suicidal thoughts factor of Concise Health Risk Tracking (CHRT) scale. After removing suicide-related item, overall depression was assessed with the Hamilton Depression Rating Scale (HAMD-17) in the EMBARC trial, and with Quick Inventory of Depressive Symptomatology Clinician-Rated version (QIDS-C) in the CO-MED and SAMS trials.

CO-MED Combining Medications to Enhance Depression Outcomes, EMBARC Establishing Moderators and Biosignatures of Antidepressant Response in Clinical Care, SAMS Suicide Assessment Methodology Study.

Do early changes in irritability with treatment predict subsequent levels of SI even after controlling for changes in overall depression?

Yes. In CO-MED, EMBARC, and SAMS trials, greater baseline-toweek-2 reduction in irritability predicted lower levels of SI from week-2-to-week-8 [ $\beta$ (SE) were $-0.08(0.03, p=0.023),-0.50(0.05$, $p<0.0001)$, and $-0.12(0.05, p=0.024)$ in CO-MED, EMBARC, and SAMS respectively] even after controlling for baseline levels of $\mathrm{SI}$, irritability, and overall depression as well as baseline-to-week-2 changes in overall depression, also see Table 3.

\section{Additional analyses}

Concurrent association between irritability and SI was significant even after controlling for levels of anxiety $(\beta=0.08-0.36$, all $p<$ 0.0001 , Supplementary Table S2) or insomnia $(\beta=0.09-0.37$, all $p<0.0001$, Supplementary Table S3) at the same visit. Furthermore, greater baseline-to-week-2 reduction in irritability predicted lower levels of SI from week-2-to-week-8 even after controlling for changes in anxiety $(\beta=-0.15$ to -0.51 , all $p<0.05$, Supplementary Table S5) or insomnia $(\beta=-0.12$ to -0.51 , all $p<0.05$, Supplementary Table S6).

Excluding the impulsivity-related item did not significantly change the correlation between irritability and SI (Pearson's $r=$ $0.28-0.74$ vs. $r=0.31-0.73$ with five-item (AST-IRR). Concurrent association between this modified scale of irritability and SI was significant even after controlling for overall depression $(\beta=$ 0.04-0.62, all $p<0.0001$, Supplementary Table S4). Similarly, greater baseline-to-week-2 reduction in this modified scale of irritability predicted lower levels of suicidality from week-2-toweek-8 even after controlling for overall depression $(\beta=-0.07$ to -0.51 , all $p<0.05$, Supplementary Table S7).

In EMBARC, higher irritability was associated with higher $\mathrm{SI}$ concurrently in both sertraline $(\beta=0.68 ; 95 \% \mathrm{Cl}: 0.62,0.74 ; p<$ $0.0001)$ and placebo $(\beta=0.61 ; 95 \% \mathrm{Cl}: 0.55,0.68 ; p<0.001)$ arms even after controlling for overall depression. Similarly, greater baseline-to-week- 2 reduction in irritability predicted lower levels of SI from week-2-to-week-8 in both sertraline $(\beta=-0.41 ; 95 \% \mathrm{Cl}$ : $-0.28,-0.54 ; p<0.0001)$ and placebo $(\beta=-0.49 ; 95 \% \mathrm{Cl}:-0.32$, $-0.65 ; p<0.0001)$ arms even after controlling for baseline-toweek-2 reduction in overall depression.

\section{DISCUSSION}

Across three separate clinical trials, we found consistent evidence linking irritability to concurrent SI. In fact, this association was stronger than that of overall depression and SI in two out of three trials. Furthermore, we found that greater reduction in irritability earlier in the course of treatment was associated with reduced levels of SI throughout the remainder of acute-phase treatment. The association between irritability and $\mathrm{SI}$ continued to be significant even when controlling for anxiety or insomnia.

Our finding that higher irritability is associated with greater SI at the same visit is consistent with the previous report by Berk et al. [10], who found that bipolar or schizoaffective patients with irritability had higher scores on the suicidality item of HAMD at index episode. Findings of this report are also consistent with studies that have linked irritability to subsequent suicidality in youths $[7,8]$. However, to our knowledge, this is the first longitudinal study in adult patients with MDD describing the association between irritability and SI throughout the course of treatment. In addition, we found that the association between irritability and SI was not accounted for by severity of other depressive symptoms, anxiety, or insomnia and that irritability may be a stronger predictor of SI than these other symptoms. Most notably, these findings were remarkably consistent across three separate samples of patients with MDD.

Findings of this report are consistent with emerging literature on the association between anger (a construct related to irritability) and suicidality. Cross-sectional studies have reported significant associations between anger and suicidality in community samples [40], including home-dwelling elderly [41], veterans [42], and patients with chronic cancer pain [43], somatoform disorders [44], and mood or anxiety disorders [45]. Similarly, longitudinal studies have also reported an association between anger and subsequent $\mathrm{Sl}$, suicide attempt, or completed suicide [46-48]. Taken together, these findings argue for careful assessment of irritability and related constructs in suicide risk assessment. However, the causes of these associations between irritability and SI remain unclear. One possible explanation could be that irritability is associated with greater propensity for aggression in response to frustration or threat (reactive aggression) $[49,50]$. Reactive aggression, in turn, has been consistently associated with suicide-related behaviors [51]. Another mechanism of potential relevance may be the hyperarousal state that is associated with irritability [52] and has been linked to suicidality in past $[53,54]$.

These findings may have clinical implications. Higher levels of irritability may alert clinicians to the risk of elevated SI. Hence, these findings add to the clinical utility of incorporating assessments of irritability in measurement-based care approach to management of depression $[22,24,55]$. This may be especially 
CO-MED Overall Depression

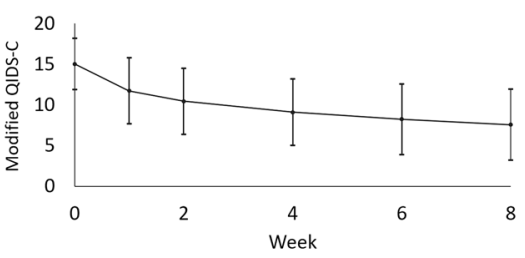

CO-MED Irritability

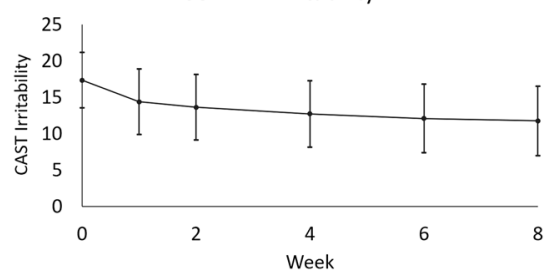

CO-MED Suicidal Ideation

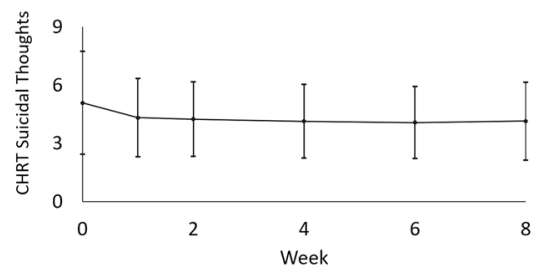

EMBARC Overall Depression

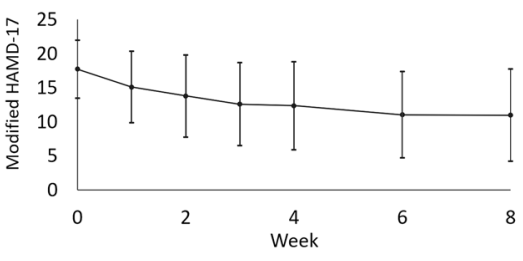

EMBARC Irritability

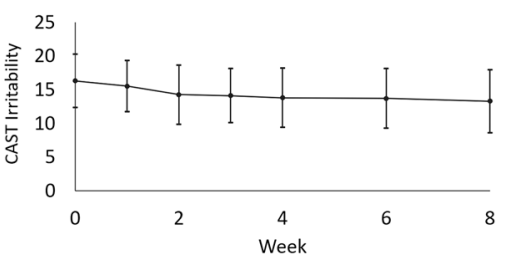

EMBARC Suicidal Ideation

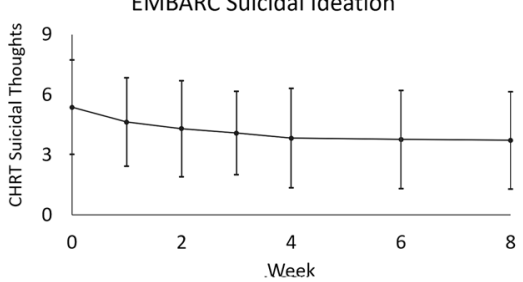

SAMS Overall Depression

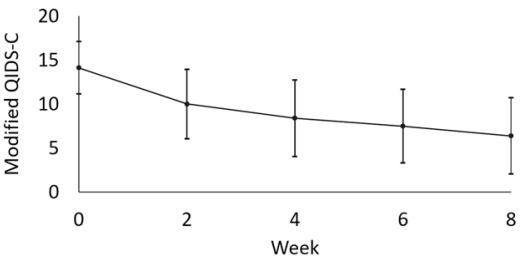

SAMS Irritability

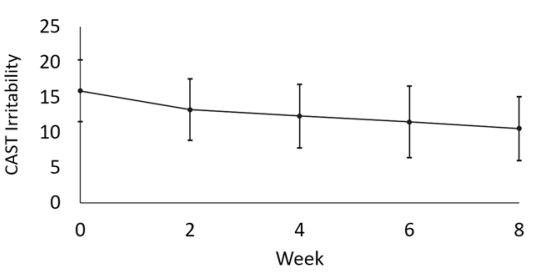

SAMS Suicidal Ideation

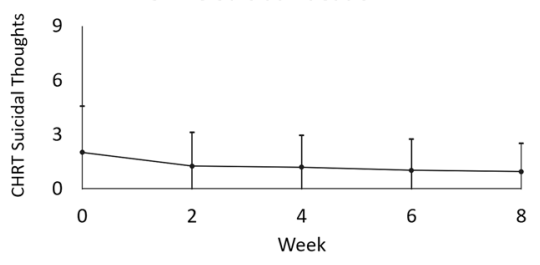

Fig. 1 Levels of overall depression, irritability, and suicidal ideation, during CO-MED, EMBARC, and SAMS trials. CO-MED is Combining Medications to Enhance Depression Outcomes $(n=665)$, EMBARC is Establishing Moderators and Biosignatures of Antidepressant Response in Clinical Care $(n=296)$, and SAMS is Suicide Assessment Methodology Study $(n=266)$. Irritability was measured with the five-item irritability domain of Concise Associated Symptom Tracking (CAST) scale. Suicidal ideation was measured with three-item suicidal thoughts factor of Concise Health Risk Tracking (CHRT) scale. After removing the suicide-related item, overall depression was assessed with the Hamilton Depression Rating Scale (modified HAMD-17) in the EMBARC trial, and with Quick Inventory of Depressive Symptomatology Clinician-Rated version (modified QIDS-C) in the CO-MED and SAMS trials. Error bars represent 1 standard deviation above and below observed means at each visit.

Table 2. Concurrent association between repeated observations of suicidal ideation with irritability, anxiety, insomnia, and overall depression in COMED, EMBARC, and SAMS trials.

\begin{tabular}{|c|c|c|c|c|c|c|}
\hline Anxiety and suicidal ideation & 0.24 & $0.19,0.29$ & 0.36 & $0.30,0.43$ & 0.20 & $0.12,0.30$ \\
\hline Insomnia and suicidal ideation & 0.18 & $0.14,0.22$ & 0.25 & $0.19,0.32$ & 0.21 & $0.14,0.29$ \\
\hline Overall depression and suicidal ideation & 0.28 & $0.24,0.33$ & 0.41 & $0.35,0.47$ & 0.23 & $0.15,0.30$ \\
\hline
\end{tabular}

important for pediatric and young adult patients where the use of antidepressants may be restricted by the black box warning by the Food and Drug Administration regarding increased risk of suicidal thoughts and behaviors with antidepressants in these patients [56]. Therefore, future studies are needed in youths to characterize the longitudinal associations between irritability and suiciderelated outcomes within the context of treatment with serotonergic antidepressants. Furthermore, there is an urgent need to develop treatment strategies that specifically target irritability. While a recent study showed that citalopram, a SSRI antidepressant, was superior to placebo in reducing severe chronic irritability in youths [57], a previous report had found worsening of irritability in a sizeable proportion (about 6\%) of patients with MDD who were treated either SSRI monotherapy or a combination of antidepressants [32].
We found that associations between irritability and SI did not differ between the two treatment arms (sertraline and placebo) of EMBARC study. We also found that the association between irritability and SI was numerically higher in EMBARC $(r=0.73)$ as compared to CO-MED $(r=0.31)$ and SAMS $(r=0.31)$ trials which may reflect the heterogeneity within the syndromic diagnosis of MDD. This may also be related to sample characteristics; participants of EMBARC were volunteers who were recruited from depression clinical research programs at four major academic medical centers whereas those of CO-MED and SAMS were treatment-seeking outpatients from community-based primary care and psychiatric practices who agreed to participate in the trial and were treated within these practices during the trial. In addition, EMBARC study had relatively extensive eligibility restrictions (related to EMBARC's emphasis on biosignature 
A

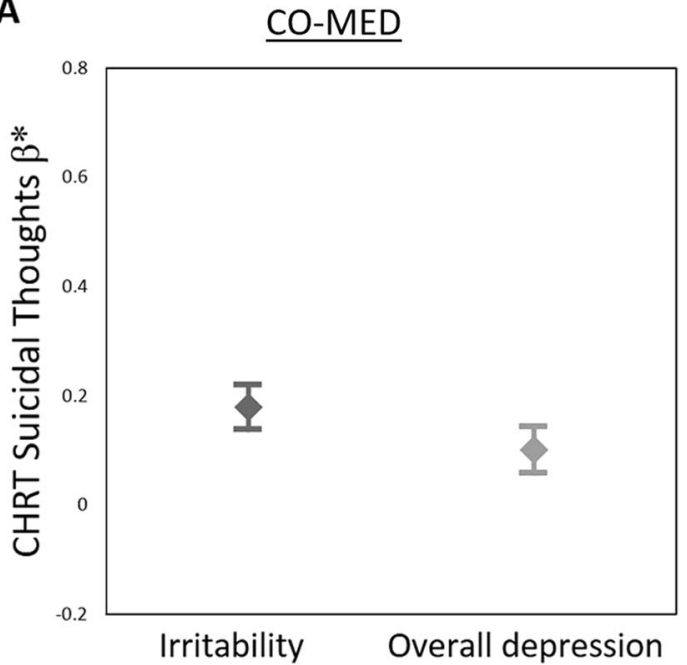

B

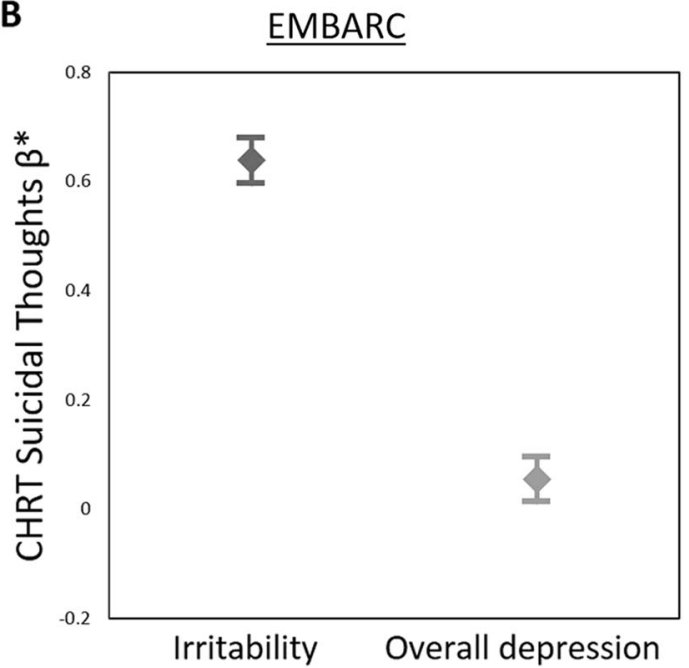

C

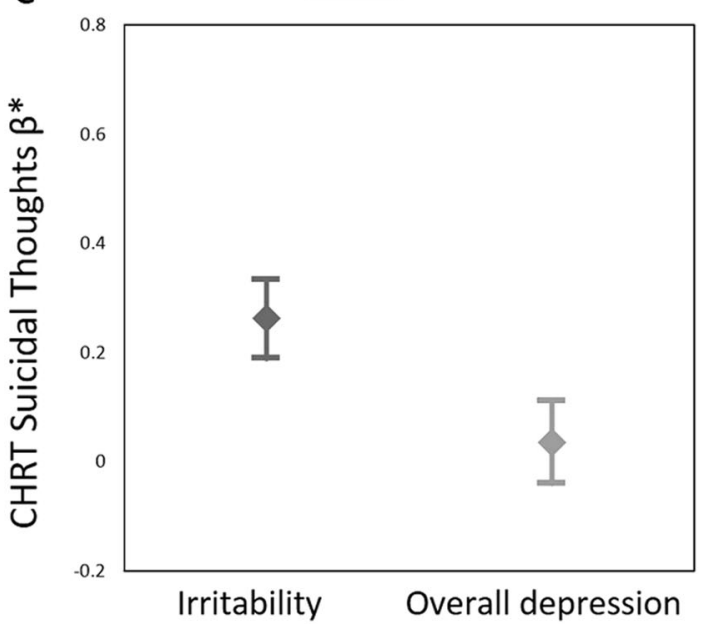

Fig. 2 Association of suicidal ideation with irritability and overall depression in CO-MED, EMBARC, and SAMS trials. * Standardized $\beta$ estimates (all symptom measures were standardized with mean of 0 and standard deviation of 1) were obtained from separate linear mixed models with Concise Health Risk Tracking (CHRT) scale suicidal thoughts factor as the dependent variable and irritability and overall depression as independent variables in Combining Medications to Enhance Depression Outcomes (CO-MED; $n=665$, a), Establishing Moderators and Biosignatures of Antidepressant Response in Clinical Care (EMBARC; $n=296$, b), and Suicide Assessment Methodology Study (SAMS; $n=266$, c). Error bars represent $95 \%$ confidence interval of the standardized $\beta$ estimates. Irritability was assessed with the five-item irritability domain of Concise Associated Symptom Tracking (CAST) scale. Suicidal ideation was assessed with three-item suicidal thoughts factor of Concise Health Risk Tracking (CHRT) scale. Overall depression was assessed with the Hamilton Depression Rating Scale in the EMBARC trial, and with Quick Inventory of Depressive Symptomatology Clinician-Rated version in the CO-MED and SAMS trials, after excluding the suicidality-related item.

Table 3. Association between levels of suicidal ideation from week-2to-week-8 in repeated-measures mixed model analyses based on early changes in irritability and overall depression.

\begin{tabular}{|c|c|c|c|c|c|}
\hline & $\beta^{*}$ & SE & $F$ value & df & $p$ value \\
\hline \multicolumn{6}{|l|}{ CO-MED } \\
\hline Baseline suicidal ideation & 0.17 & 0.01 & 251.98 & 1,539 & $<0.0001$ \\
\hline Baseline irritability & 0.03 & 0.03 & 1.02 & 1,542 & 0.31 \\
\hline Baseline overall depression & 0.10 & 0.03 & 8.85 & 1,546 & 0.003 \\
\hline $\begin{array}{l}\text { Baseline-to-week-2 change in } \\
\text { irritability }\end{array}$ & -0.08 & 0.03 & 5.19 & 1,534 & 0.023 \\
\hline $\begin{array}{l}\text { Baseline-to-week-2 change in } \\
\text { overall depression }\end{array}$ & -0.13 & 0.03 & 15.07 & 1,548 & 0.0001 \\
\hline \multicolumn{6}{|l|}{ EMBARC } \\
\hline Baseline suicidal ideation & 0.30 & 0.05 & 36.18 & 1,216 & $<0.0001$ \\
\hline Baseline irritability & 0.41 & 0.06 & 50.85 & 1,216 & $<0.0001$ \\
\hline Baseline overall depression & 0.02 & 0.05 & 0.11 & 1,216 & 0.74 \\
\hline $\begin{array}{l}\text { Baseline-to-week-2 change in } \\
\text { irritability }\end{array}$ & -0.50 & 0.05 & 85.87 & 1,214 & $<0.0001$ \\
\hline $\begin{array}{l}\text { Baseline-to-week-2 change in } \\
\text { overall depression }\end{array}$ & -0.03 & 0.05 & 0.44 & 1,215 & 0.51 \\
\hline \multicolumn{6}{|l|}{ SAMS } \\
\hline Baseline suicidal ideation & 0.42 & 0.04 & 87.57 & 1,191 & $<0.0001$ \\
\hline Baseline irritability & 0.08 & 0.05 & 2.27 & 1, 192 & 0.13 \\
\hline Baseline overall depression & -0.00 & 0.02 & 0.07 & 1,186 & 0.79 \\
\hline $\begin{array}{l}\text { Baseline-to-week-2 change in } \\
\text { irritability }\end{array}$ & -0.12 & 0.05 & 5.22 & 1,187 & 0.024 \\
\hline $\begin{array}{l}\text { Baseline-to-week-2 change in } \\
\text { overall depression }\end{array}$ & -0.07 & 0.05 & 1.59 & 1,188 & 0.21 \\
\hline
\end{tabular}

$\beta^{*}$ is estimate obtained from solution of fixed effects from mixed model analyses with suicidal ideation as the dependent variable (all symptom measures were standardized with mean of 0 and standard deviation of 1). All analyses included visit, age, sex, race, and ethnicity as covariates SE standard error, df is degrees of freedom. CO-MED Combining Medications to Enhance Depression Outcomes, EMBARC Establishing Moderators and Biosignatures of Antidepressant Response in Clinical Care, SAMS Suicide Assessment Methodology.

\section{Limitations}

The assessment of irritability was restricted to a self-report measure which differs from studies in youths where it is common to include assessments from parent/teacher(s) [18]. The measure 
of irritability in this report is based on factor analysis of a scale that was initially developed for measurement of depression-associated symptoms and includes an impulsivity-related item. However, the exclusion of this impulsivity-related item did not alter the association between irritability and SI. None of the trials reported were powered a priori to evaluate the association between irritability and $\mathrm{SI}$, thus these findings should be considered preliminary and need to be validated in prospective studies. To ensure uniformity across trials in this report, we restricted the assessment of overall depression to a clinician-rated scale while measures of SI and irritability were patient self-report. However, clinician and patient-rated measures of overall depression have been shown to reflect the same symptom severity and change constructs allowing for conversion from one scale to another [37]. Findings of our study are also limited by eligibility criteria, which excluded pediatric patients and may not be generalizable to this vulnerable population. An additional limitation includes measurement of SI with a self-report scale, which did not collect information about suicidal behaviors and/or mortality. Thus, future studies are needed to evaluate whether irritability is a modifiable risk factor of future suicidal behaviors and suicide-related mortality. Irritability, overall depression, anxiety, and insomnia are related constructs. Thus, future studies are needed to understand the shared versus unique contributions of these in predicting SI. Finally, none of these studies included a treatment that specifically targets irritability. Thus, prospective studies of irritability-specific treatments are needed to demonstrate that reduction in irritability is associated with subsequent improvement of SI [26].

\section{CONCLUSION}

Higher levels of irritability are associated with higher levels of concurrent SI throughout the duration of treatment, and reduction in irritability earlier in course of treatment predicts lower levels of SI subsequently. Taken together, these findings add to the emerging literature on the importance of carefully assessing and targeting irritability in adults with MDD.

\section{FUNDING AND DISCLOSURES}

Combined Medications to Enhance Depression Outcomes (COMED) and Suicide Assessment and Methodology Study (SAMS) were funded by NIMH under contract N01 MH-90003 to the University of Texas Southwestern Medical Center at Dallas (PIs: Rush and MT). The Establishing Moderators and Biosignatures of Antidepressant Response in Clinical Care (EMBARC) study was supported by NIMH grants U01MH092221 (PI: MT) and U01MH092250 (PIs: McGrath, Parsey, and Weissman). This work was also funded in part by the Hersh Foundation (PI: MT). Forest Pharmaceuticals, GlaxoSmithKline, Organon, and Wyeth Pharmaceuticals provided medications for this CO-MED trial at no cost. The content of this publication does not necessarily reflect the views or policies of the US Department of Health and Human Services, nor does mention of trade names, commercial products, or organizations imply endorsement by the US government. NIMH had no role in the drafting or review of the paper or in the collection or analysis of the data.

$\mathrm{AM}, \mathrm{CCF}, \mathrm{EL}$, and $\mathrm{KK}$ have no conflicts to report. MKJ has received contract research grants from Acadia Pharmaceuticals and Janssen Research \& Development, and honoraria for CME presentations from North American Center for Continuing Medical Education and Global Medical Education. AS receives royalties from Oxford and Cambridge University Press. MT has served as an adviser or consultant for Abbott Laboratories, Abdi Ibrahim, Akzo (Organon Pharmaceuticals), Alkermes, AstraZeneca, Axon Advisors, Bristol-Myers Squibb, Cephalon, Cerecor, CME Institute of Physicians, Concert Pharmaceuticals, Eli Lilly, Evotec, Fabre Kramer
Pharmaceuticals, Forest Pharmaceuticals, GlaxoSmithKline, Janssen Global Services, Janssen Pharmaceutica Products, Johnson \& Johnson PRD, Libby, Lundbeck, Meade Johnson, MedAvante, Medtronic, Merck, Mitsubishi Tanabe Pharma Development America, Naurex, Neuronetics, Otsuka Pharmaceuticals, Pamlab, Parke-Davis Pharmaceuticals, Pfizer, PgxHealth, Phoenix Marketing Solutions, Rexahn Pharmaceuticals, Ridge Diagnostics, Roche Products, Sepracor, Shire Development, Sierra, SK Life and Science, Sunovion, Takeda, Tal Medical/Puretech Venture, Targacept, Transcept, VantagePoint, Vivus, and Wyeth-Ayerst Laboratories; he has received grants or research support from the Agency for Healthcare Research and Quality, Cyberonics, NARSAD, NIDA, and NIMH.

The authors thank the clinical staff at each clinical site for their assistance with this project; all of the study participants; and Georganna Carlock, B.A., Eric Nestler, M.D., Ph.D., and Carol A. Tamminga, M.D., for administrative support. NIMH, Hersh Foundation.

\section{AUTHOR CONTRIBUTIONS}

Study design: MHT. Obtained funding: MT. Data acquisition: MKJ and MT. Data analysis: AM. Data interpretation: MKJ, AM, CCF, KK, AS, EL, and MT. Drafting of the work: MKJ. Revising paper critically for important intellectual content: MKJ, AM, CCF, KK, AS, Leibenluft, MT. Final approval: MKJ, AM, CCF, KK, AS, EL, and MT. The design of CO-MED, EMBARC and SAMS was led by MT. MKJ and MT contributed substantially to the acquisition of data. AM completed the data analysis. MKJ, AM, CCF, KK, AS, EL, and MT contributed to the interpretation of data. MKJ drafted the initial paper and all authors revised paper critically for important intellectual content. All authors provided the final approval to the submitted version and agree to be accountable for all aspects of the work in ensuring that questions related to the accuracy or integrity of any part of the work are appropriately investigated and resolved.

\section{ADDITIONAL INFORMATION}

Supplementary Information accompanies this paper at (https://doi.org/10.1038/ s41386-020-0769-x).

Publisher's note Springer Nature remains neutral with regard to jurisdictional claims in published maps and institutional affiliations.

\section{REFERENCES}

1. Curtin $\mathrm{S}$, Hedegaard $\mathrm{H}$. Suicide rates for females and males by race and ethnicity: United States, 1999 and 2017. NCHS Health E-Stat; 2019. https://www.cdc.gov/ nchs/data/hestat/suicide/rates_1999_2017.pdf. Accessed 11 Mar 2020.

2. Brown GK, Beck AT, Steer RA, Grisham JR. Risk factors for suicide in psychiatric outpatients: a 20-year prospective study. J Consul Clin Psychol. 2000;68:371-7.

3. Hawton K, Casanas ICC, Haw C, Saunders K. Risk factors for suicide in individuals with depression: a systematic review. J Affect Disord. 2013;147:17-28.

4. Hawton K, Sutton L, Haw C, Sinclair J, Harriss L. Suicide and attempted suicide in bipolar disorder: a systematic review of risk factors. J Clin Psychiatry. 2005;66: 693-704.

5. Orri M, Perret LC, Turecki G, Geoffroy MC. Association between irritability and suicide-related outcomes across the life-course. Systematic review of both community and clinical studies. J Affect Disord. 2018;239:220-33.

6. Conner KR, Meldrum S, Wieczorek WF, Duberstein PR, Welte JW. The association of irritability and impulsivity with suicidal ideation among 15- to 20-year-old males. Suicide Life-Threat Behav. 2004;34:363-73.

7. Orri M, Galera C, Turecki G, et al. Association of childhood irritability and depressive/anxious mood profiles with adolescent suicidal ideation and attempts. JAMA Psychiatry 2018;75:465-73.

8. Pickles A, Aglan A, Collishaw S, Messer J, Rutter M, Maughan B. Predictors of suicidality across the life span: The Isle of Wight study. Psychological Med 2009:40:1453-66.

9. Kuba T, Yakushi T, Fukuhara $\mathrm{H}$, et al. Suicide-related events among child and adolescent patients during short-term antidepressant therapy. Psychiatry Clin Neurosci. 2011;65:239-45.

10. Berk L, Hallam KT, Venugopal K, et al. Impact of irritability: a 2-year observational study of outpatients with bipolar I or schizoaffective disorder. Bipolar Disord 2017;19:184-97. 
11. Melhem NM, Porta G, Oquendo MA, et al. Severity and variability of depression symptoms predicting suicide attempt in high-risk individuals. JAMA Psychiatry 2019;76:603-13.

12. Boudreaux ED, Larkin $\mathrm{C}$, Kini N, et al. Predictive utility of an emergency department decision support tool in patients with active suicidal ideation. Psychol Serv. 2018;15:270-8.

13. McGarry A, McDermott MP, Kieburtz K, et al. Risk factors for suicidality in Huntington disease: An analysis of the 2CARE clinical trial. Neurology 2019;92: e1643-e1651.

14. Perlis $\mathrm{RH}$, Fava $\mathrm{M}$, Trivedi $\mathrm{MH}$, et al. Irritability is associated with anxiety and greater severity, but not bipolar spectrum features, in major depressive disorder. Acta Psychiatr Scand. 2009;119:282-9.

15. Pendse B, Westrin A, Engstrom G. Temperament traits in seasonal affective disorder, suicide attempters with non-seasonal major depression and healthy controls. J Affect Disord. 1999;54:55-65.

16. Azorin JM, Kaladjian A, Besnier N, et al. Suicidal behaviour in a French Cohort of major depressive patients: characteristics of attempters and nonattempters. J Affect Disord. 2010;123:87-94.

17. Akiskal HS, Benazzi F. Does the FDA proposed list of possible correlates of suicidality associated with antidepressants apply to an adult private practice population? J Affect Disord. 2006;94:105-10.

18. Brotman MA, Kircanski K, Stringaris A, Pine DS, Leibenluft E. Irritability in youths: a translational model. Am J Psychiatry. 2017;174:520-32.

19. AP Association. Diagnostic and statistical manual of mental disorders (DSM- $5^{\circ}$ ). American Psychiatric Pub; Arlington, VA, 2013.

20. Fava M, Hwang I, Rush AJ, Sampson N, Walters EE, Kessler RC. The importance of irritability as a symptom of major depressive disorder: results from the National Comorbidity Survey Replication. Mol Psychiatry 2010;15:856.

21. Perlis RH, Uher R, Ostacher $M$, et al. Association between bipolar spectrum features and treatment outcomes in outpatients with major depressive disorderbipolar spectrum features and treatment outcomes in MDD. JAMA Psychiatry 2011;68:351-60.

22. Jha MK, Minhajuddin A, South C, Rush AJ, Trivedi MH. Irritability and its clinical utility in major depressive disorder: prediction of individual-level acute-phase outcomes using early changes in irritability and depression severity. Am J Psychiatry. 2019;176:358-66.

23. Pine DanielS. Heterogeneity in major depressive disorder: lessons from developmental research on irritability. Am J Psychiatry. 2019;176:331-2.

24. Jha MK, Grannemann BD, Trombello JM, et al. A structured approach to detecting and treating depression in primary care: VitalSign6 Project. Ann Fam Med. 2019;17:326-35.

25. Kircanski K, White LK, Tseng WL, et al. A latent variable approach to differentiating neural mechanisms of irritability and anxiety in youth. JAMA Psychiatry 2018;75:631-9.

26. McCall WV, Benca RM, Rosenquist PB, et al. Reducing Suicidal Ideation Through Insomnia Treatment (REST-IT): a randomized clinical trial. Am J Psychiatry. 2019;176:957-65.

27. Rush AJ, Trivedi MH, Stewart JW, et al. Combining medications to enhance depression outcomes (CO-MED): acute and long-term outcomes of a single-blind randomized study. Am J Psychiatry. 2011;168:689-701.

28. Trivedi MH, McGrath PJ, Fava M, et al. Establishing moderators and biosignatures of antidepressant response in clinical care (EMBARC): Rationale and design. J Psychiatr Res. 2016;78:11-23.

29. Trivedi $M H$, South $C$, Jha $M K$, et al. A novel strategy to identify placebo responders: prediction index of clinical and biological markers in the EMBARC Trial. Psychother Psychosom. 2018;87:285-95.

30. Trivedi MH, Wisniewski SR, Morris DW, et al. Concise health risk tracking scale: a brief self-report and clinician rating of suicidal risk. J Clin Psychiatry. 2011;72: 757-64.

31. Trivedi MH, Wisniewski SR, Morris DW, et al. Concise associated symptoms tracking scale: a brief self-report and clinician rating of symptoms associated with suicidality. J Clin Psychiatry. 2011;72:765-74.

32. Jha MK, Minhajuddin A, South C, Rush AJ, Trivedi MH. Worsening anxiety, irritability, insomnia, or panic predicts poorer antidepressant treatment outcomes: clinical utility and validation of the Concise Associated Symptom Tracking (CAST) Scale. Int J Neuropsychopharmacol. 2018;21:325-32.

33. Minhajuddin A, Jha MK, Chin Fatt C, Trivedi MH. Psychometric properties of Concise Associated Symptom Tracking (CAST) scale and validation of its clinical utility in patients with major depressive disorder: findings from the EMBARC study. Psychiatr Res Clin Pract. 2020. https://doi.org/10.1176/appi.prcp.20190041.

34. Williams JB. A structured interview guide for the Hamilton Depression Rating Scale. Arch Gen Psychiatry. 1988;45:742-7.

35. Hamilton M. A rating scale for depression. J Neurol Neurosurg Psychiatry. 1960;23:56-62.

36. Rush AJ, Trivedi MH, Ibrahim HM, et al. The 16-Item Quick Inventory of Depressive Symptomatology (QIDS), clinician rating (QIDS-C), and self-report (QIDS-SR): a psychometric evaluation in patients with chronic major depression. Biol Psychiatry 2003;54:573-83.

37. Vittengl JR, Clark LA, Kraft D, Jarrett RB. Multiple measures, methods, and moments: a factor-analytic investigation of change in depressive symptoms during acute-phase cognitive therapy for depression. Psychol Med. 2005;35: 693-704.

38. Trombello JM, Killian MO, Grannemann BD, et al. The Concise Health Risk Tracking-Self Report: Psychometrics within a placebo-controlled antidepressant trial among depressed outpatients. J Psychopharmacol. 2019;33:185-93.

39. Jha MK, Minhajuddin A, Greer TL, Carmody T, Rush AJ, Trivedi MH. Early improvement in psychosocial function predicts longer-term symptomatic remission in depressed patients. PLoS ONE. 2016;11:e0167901.

40. Jang JM, Park Jl, Oh KY, et al. Predictors of suicidal ideation in a community sample: roles of anger, self-esteem, and depression. Psychiatry Res. 2014;216: 74-81.

41. Zhang $W$, Ding $H$, Su $P$, et al. Prevalence and risk factors for attempted suicide in the elderly: a cross-sectional study in Shanghai, China. Int Psychogeriatr. 2017;29: 709-15.

42. Wilks CR, Morland LA, Dillon KH, et al. Anger, social support, and suicide risk in U. S. military veterans. J Psychiatr Res. 2019;109:139-44.

43. Racine M, Sanchez-Rodriguez E, Galan S, et al. Factors associated with suicidal ideation in patients with chronic non-cancer pain. Pain Med. 2017;18:283-93.

44. Kampfer N, Staufenbiel S, Wegener I, et al. Suicidality in patients with somatoform disorder-the speechless expression of anger? Psychiatry Res. 2016;246: 485-91.

45. Stringer B, van Meijel B, Eikelenboom M, et al. Recurrent suicide attempts in patients with depressive and anxiety disorders: the role of borderline personality traits. J Affect Disord. 2013;151:23-30.

46. Hogstedt $C$, Forsell $Y$, Hemmingsson $T$, Lundberg I, Lundin A. Psychological symptoms in late adolescence and long-term risk of suicide and suicide attempt. Suicide Life-Threat Behav. 2018;48:315-27.

47. Start AR, Allard Y, Adler A, Toblin R. Predicting suicide ideation in the military: the independent role of aggression. Suicide Life-Threat Behav. 2019;49:444-54.

48. Ducasse D, Jaussent I, Guillaume $S$, et al. Affect lability predicts occurrence of suicidal ideation in bipolar patients: a two-year prospective study. Acta Psychiatr Scand. 2017;135:460-9.

49. Leibenluft E. Irritability in children: what we know and what we need to learn. World Psychiatry. 2017:16:100-1.

50. Brotman MA, Kircanski K, Leibenluft E. Irritability in children and adolescents. Annu Rev Clin Psychol. 2017;13:317-41.

51. Hartley CM, Pettit JW, Castellanos D. Reactive aggression and suicide-related behaviors in children and adolescents: a review and preliminary meta-analysis. Suicide Life-Threat Behav. 2018:48:38-51.

52. Toohey MJ, DiGiuseppe R. Defining and measuring irritability: construct clarification and differentiation. Clin Psychol Rev. 2017;53:93-108.

53. Stanley IH, Rogers ML, Hanson JE, Gutierrez PM, Joiner TE. PTSD symptom clusters and suicide attempts among high-risk military service members: a three-month prospective investigation. J Consult Clin Psychol. 2019;87:67-78.

54. Dolsen MR, Cheng P, Arnedt JT, et al. Neurophysiological correlates of suicidal ideation in major depressive disorder: Hyperarousal during sleep. J Affect Disord. 2017;212:160-6.

55. Trivedi MH, Jha MK, Kahalnik F, et al. VitalSign(6): a primary care first (PCP-First) model for universal screening and measurement-based care for depression. Pharmaceuticals. 2019;12:71. https://doi.org/10.3390/ph12020071.

56. Friedman RA. Antidepressants' black-box warning-10 years later. N Engl J Med. 2014;371:1666-8.

57. Towbin K, Vidal-Ribas $P$, Brotman MA, et al. A double-blind randomized placebo-controlled trial of citalopram adjunctive to stimulant medication in youth with chronic severe irritability. J Am Acad Child Adolesc Psychiatry. 2019:59:350-61. 